\title{
ROMANIAN
}

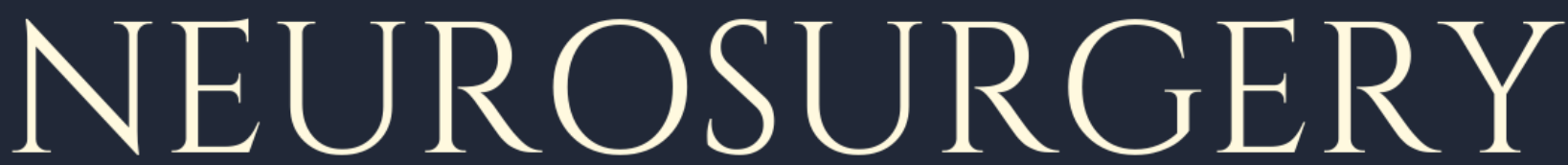

\author{
Vol. XXXV | No. $1 \quad$ March 2021
}

\section{Volumetric threshold of pituitary macroadenoma as a predictor to visual impairment. Clinical correlation}

\author{
Mohamed Elsherbini, \\ Mahmoud Saad, \\ Mohamed Deniwar
}




\title{
Volumetric threshold of pituitary macroadenoma as a predictor to visual impairment. Clinical correlation
}

\author{
Mohamed Elsherbini, Mahmoud Saad, Mohamed Deniwar
}

\author{
Mansoura University Hospital, Elgomhoreya St., Mansoura, EGYPT
}

\begin{abstract}
Purpose: In this study, we aimed at correlating the curve of visual impairment against pituitary macroadenoma size.

Method: In this study, we retrospectively analyzed the visual correlation between the volume and dimensions of pituitary adenoma and the degree of visual impairment on patients' examination. 35 patients with no history of eye or refraction disorder were included in the study. Spearman correlation test was used to validate the correlation.

Results: $57 \%$ of pituitary macroadenoma patients suffer from visual impairment, even if not the primary presentation. Macroadenomas greater than $5 \mathrm{~cm}^{3}$ in volume and/or $2 \mathrm{~cm}$ height are more likely to cause various degrees of visual impairment, this correlation is not linear beyond these values.

Conclusion: Visual impairment due to pituitary adenomas is more frequent than the presentation, the threshold volume is $5 \mathrm{~cm}^{3}$ and height is $2 \mathrm{~cm}$, bigger adenomas behaviour is more multifactorial than the only size.
\end{abstract}

\section{INTRODUCTION}

Pituitary gland lesions are commonly associated with visual manifestations at different levels, either as the primary presentation, or as a finding during neurological examination. These manifestations are explained by the intimate relationship between the pituitary gland and the visual pathway.

The pituitary gland is covered superiorly by the diaphragma sellae; a fibrous dural layer with a small opening called the diaphragmatic hiatus through which the infundibulum courses. The suprasellar cistern is located above the diaphragma sellae and separates it from the optic chiasm. The suprasellar cistern is first compressed by pituitary tumors before their growth proceeds to compress the optic chiasm. The size of the pituitary gland varies along life and differs from male to female; at birth it weights around $100 \mathrm{mg}$, while in adulthood it reaches up to $500 \mathrm{mg}$ with tendency to be bigger in females especially during puberty, pregnancy and lactation. In adults it measures around 8-10 $\mathrm{mm}$ and reaches $12 \mathrm{~mm}$ in females during lactation. (Cox and Elster 1991).

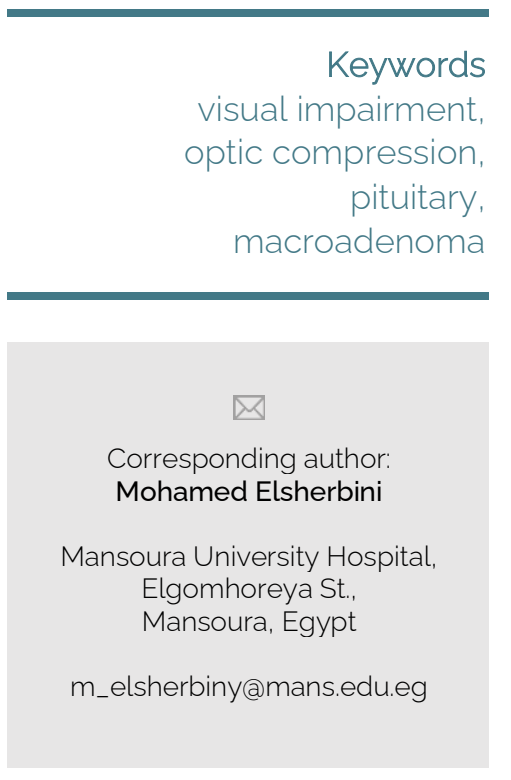

Copyright and usage. This is an Open Access article, distributed under the terms of the Creative Commons Attribution Non-Commercial No Derivatives License (https://creativecommons org/licenses/by-nc-nd/4.0/) which permits noncommercial re-use, distribution, and reproduction in any medium, provided the original work is unaltered and is properly cited.

The written permission of the Romanian Society of Neurosurgery must be obtained for commercial re-use or in order to create a derivative work.

ISSN online 2344-4959 (c) Romanian Society of Neurosurgery

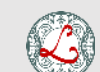

First published March 2021 by London Academic Publishing wwww.lapub.co.uk 
Pituitary adenomas, when increase in size, may lead to visual impairment via direct optic pathway compression which leads to neuronal injury via blocking axonal conduction, blocking axoplasmic flow or/and demyelination. (Munoz Negrete and Rebolleda 2002) Chronic optic pathway compression leads to optic atrophy which is irreversible, this is more noted in non-functioning pituitary adenoma patients since secretory lesions tend to present earlier by hormonal imbalance. Jagannathan, Dumont et al. 2005).

Direct optic pathway compression, though a primitive explanation, is not the only mechanism for visual impairment in patients with pituitary macroadenoma. The course of visual deterioration in relation to volume and height of the tumor is an important key to understanding the pathological process since the size of the tumor is the best predictor for visual recovery. (Monteiro, Zambon et al. 2010).

\section{PATIENTS AND METHODS}

\section{Patients data}

Data of patients admitted to Mansoura University Hospital with sellar lesion between 2017 and 2019 were reviewed and analyzed. Only patients with lesions larger than $10 \mathrm{~mm}$ in at least one dimension were included, patients with incomplete medical reports were excluded.

\section{Visual acuity}

Visual acuity is recorded in decimal method where values are obtained on snellen's chart, while severe visual impairment values are given according to Grover et (Grover, Fishman et al. 1999) al ; 0.0025 for CF "counting fingers", 0.002 for HM "hand movement", 0.0016 for "light perception" (LP), and 0.0013 for NLP "no perception of light". Values from both eyes are summed giving each patient a single value representing visual acuity in both eyes. Patients with history of eye surgeries and/or other local eye pathologies were excluded.

\section{Measurement}

Dimensions of the lesions are measured on MRI T1 with contrast axial, coronal and sagittal to obtain largest horizontal, vertical and AP diameters respectively. The volume of the tumor was calculated using the traditional geometric formula; $1 / 2$ (length $X$ width $X$ height), patients with recurrent lesions were excluded since according to Chi-Cheng Chuanf (Chuang, Lin et al. 2017) the traditional formula is not applicable for recurrent cases while it proves high degree of accuracy for primary lesions.

\section{Pathology}

Lesions were classified into functioning and nonfunctioning according to patients' pre-operative serum hormonal levels. Histopathological examination reports were reviewed, patients with lesions other than pituitary adenomas were excluded.

\section{Statistical analysis}

Spearman correlation test was used to compare visual acuity values to adenoma volume and each dimension individually. Prism GraphPad version 5 software was used to perform statistical analysis.

\section{RESULTS}

35 patients were included in the study, 18 of them were males (51\%), mean age was 46.3 years which ranged from 23 to 75 years. Most common primary presentation is hormonal related symptoms, collectively, in 15 patients, followed headache in 13 patients, while visual impairment was the primary presentation for only 7 patients (20\%). (Table 1 ).

\begin{tabular}{|c|c|c|}
\hline & & Number, \% \\
\hline $\begin{array}{r}\text { Gend } \\
- \\
-\end{array}$ & $\begin{array}{l}\text { Male } \\
\text { Female }\end{array}$ & $\begin{array}{l}18(51.4 \%) \\
17\end{array}$ \\
\hline $\begin{array}{r}\text { Clinic } \\
- \\
- \\
-\end{array}$ & $\begin{array}{l}\text { resentation: } \\
\text { Endocrinal } \\
\text { Headache } \\
\text { Visual }\end{array}$ & $\begin{array}{l}15(42.9 \%) \\
13(37.1 \%) \\
7(20 \%)\end{array}$ \\
\hline $\begin{array}{r}\text { Visua } \\
- \\
-\end{array}$ & $\begin{array}{l}\text { uity: } \\
\text { Intact } \\
\text { Mean visual acuity }\end{array}$ & $\begin{array}{l}7(20 \%) \\
0.77\end{array}$ \\
\hline $\begin{array}{r}\text { Tumo } \\
- \\
- \\
-\end{array}$ & $\begin{array}{l}\text { ze: } \\
\text { Min } \\
\text { Max } \\
\text { Mean }\end{array}$ & $\begin{array}{l}0.6 \mathrm{~cm}^{3} \\
40 \mathrm{~cm}^{3} \\
12.3 \mathrm{~cm}^{3}\end{array}$ \\
\hline $\begin{array}{r}\text { Patho } \\
- \\
- \\
- \\
-\end{array}$ & $\begin{array}{l}\text { y: } \\
\text { Non-secretory } \\
\text { GH secreting } \\
\text { Acromegaly } \\
\text { Impotence } \\
\text { Prolactin secreting } \\
\text { Amenorrhea } \\
\text { Accidentally } \\
\text { discovered }\end{array}$ & $\begin{array}{l}16(45.7 \%) \\
7(20 \%) \\
6 \\
1 \\
10(28.6 \%) \\
4 \\
4\end{array}$ \\
\hline
\end{tabular}




\begin{tabular}{|l|l|}
\hline \multicolumn{1}{|l|}{$\begin{array}{l}\text { Galactorrhea } \\
\text { Impotence } \\
\text { ACTH secreting } \\
\text { (Cushing) }\end{array}$} & 1 \\
\hline
\end{tabular}

Visual impairment was the secondary complaint in 13 patients $(37 \%)$ and on examination only 7 patients (20\%) showed intact visual acuity (2/2).

19 adenomas were secretory, 15 of these patients' primary presentation was hormonal related symptoms and 4 were accidentally discovered during pre-operative lab assessment. Acromegaly was the most common hormonal presentation in 6 patients (31.5\%), amenorrhea in 4, Cushing in 2, male sexual dysfunction in 2 and galactorrhea in one patient. Pathologically, among the 19 secretory adenomas prolactin secreting was of the highest incidence in 10 patients, GH secreting in 7 and ACTH secreting in 2 patients. Male sexual dysfunction was the primary presentation for both Prolactin and GH secreting adenomas, one patient each.

Visual acuity mean is 0.77 for the sum of both eyes, ranged from No PL in one eye to intact vision (2). Mean tumor volume was $13.3 \mathrm{~cm}^{3}$, ranged from 0.6 to $40 \mathrm{~cm}^{3}$. Mean AP diameter, width and height were $2.57,2.65$ and $2.86 \mathrm{~cm}$ respectively.

According to spearman correlation test, visual acuity impairment is negatively correlated to lesions greater than $5 \mathrm{cc}$ in volume $(r-0.67, \mathrm{P}<0.001$, Fig 1$)$ and greater than $2 \mathrm{~cm}$ in height $(r-0.6, P<0.001$, Fig 2 ), beyond these values visual acuity impairment was not in a linear relationship neither to tumor volume nor height.

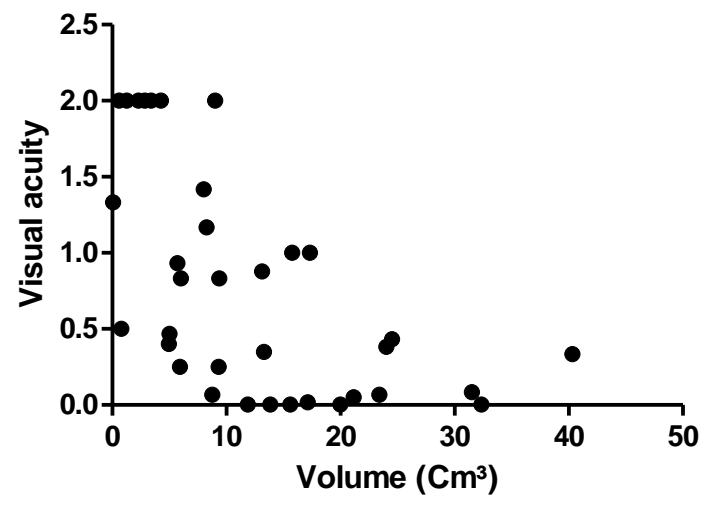

Figure 1. Representing visual acuity to tumor volume $\left(\mathrm{Cm}^{3}\right)$ correlation, showing just 2 patients with visual impairment with lesions less than $5 \mathrm{~cm}^{3}$, while strong negative correlation right to the $5 \mathrm{~cm}^{3}$ value $(r-0.67)$, but not linear.

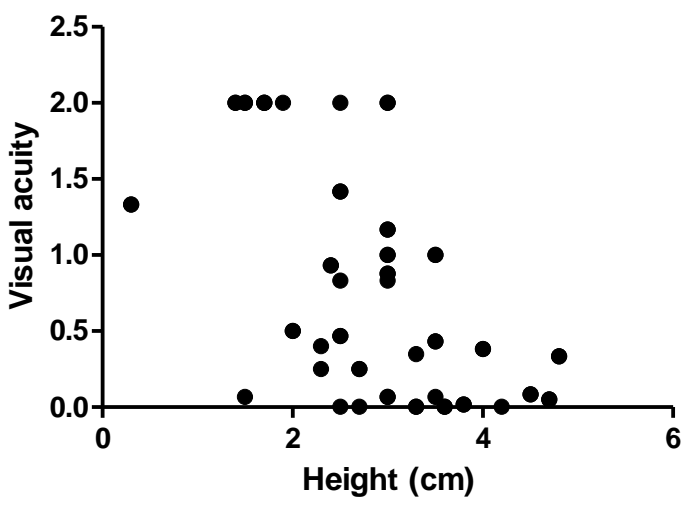

Figure 2. Representing visual acuity to tumor height $(\mathrm{Cm})$ correlation, showing just 2 patients with visual impairment with lesions less than $2 \mathrm{~cm}$, while strong negative correlation right to the $2 \mathrm{~cm}$ value $(r-0.6)$, but not linear.

\section{ILLUSTRATIVE CASES}

\section{Case 1}

41 years old male presented with acromegalic features and sexual dysfunction. Hormonal assay showed elevated serum growth hormone level. Radiologically detected $3 \times 3.5 \times 3 \mathrm{~cm}$ lesion with severe chiasm displacement and stretch, Fig 3 . Visual acuity was 0.67 and 0.33 for right and left eyes respectively.

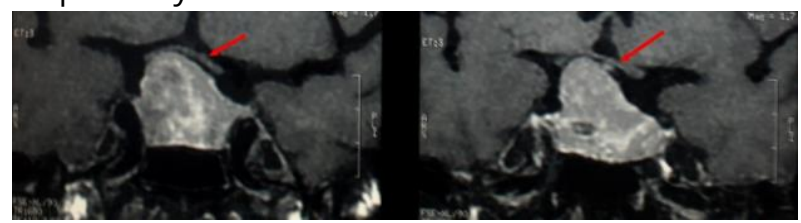

Figure 3. Case 1, Coronal MRI with contrast showing $3 \times 3.5 \times 3$ $\mathrm{cm}$ lesion with severe chiasm displacement and stretch (Red arrow).

\section{Case 2}

33 years old female lady presented with typical Cushing syndrome, elevated serum cortisol, no significant visual affection, MRI with contrast showed pituitary lesion $1.1 \times 0.5 \times 0.3 \mathrm{~cm}$ with no chiasm compression, Fig 4. Typical hormonal presentation prior optic compression.

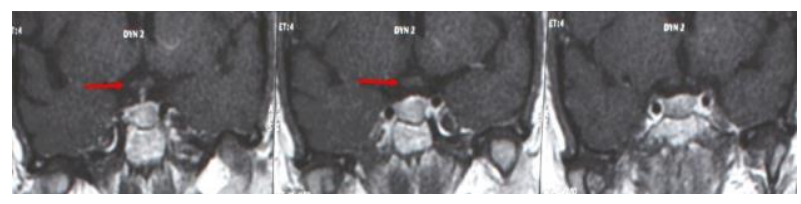

Figure 4. Case 2, Coronal MRI with contrast showing lesion 1.1 X $0.5 \times 0.3 \mathrm{~cm}$ with no chiasm compression (Red arrow). 


\section{Case 3}

55 years old post-menopausal lady presented with visual impairment; hormonal assay showed elevated serum prolactin which was not the primary presentation as the patient was postmenopausal; amenorrhea. Lesion dimensions were $2.5 \times 3.8 \times 2.5$ $\mathrm{cm}$ with severe chiasmatic compression and corresponding visual acuity NPL and HM in right and left eye respectively, Fig 5.

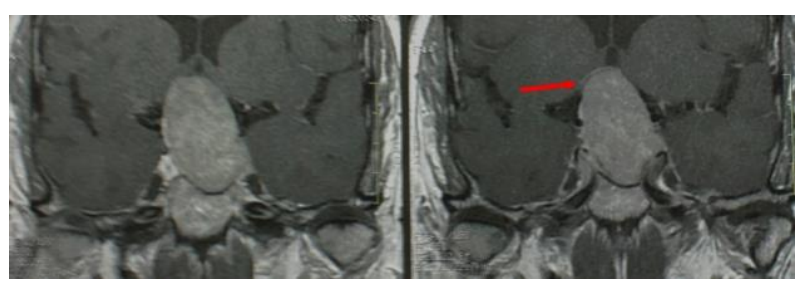

Figure 5. Case 3, Coronal MRI with contrast showing lesion 2.5 $\times 3.8 \times 2.5 \mathrm{~cm}$ with severe chiasmatic compression and stretch.

\section{Case 4}

40 years old lady presented with visual impairment, examination showed visual acuity of $\mathrm{HM}$ and $\mathrm{CF}$ in right and left eye respectively. Dimensions of the lesion in MRI were $2 \times 2.2 \times 2.7 \mathrm{~cm}$ with severe optic compression, Fig 6.

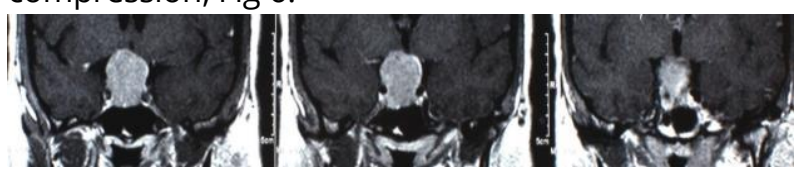

Figure 6. Case 4, Coronal MRI with contrast showing a $2 \times 2.2 \times 2.7 \mathrm{~cm}$ lesion.

\section{DISCUSSION}

Pituitary adenomas are the most common tumor of the sellar region representing $10-15 \%$ of all intracranial neoplasm, they are classified according to size into microadenomas and macroadenomas; greater than $10 \mathrm{~mm}$ (Asa and Ezzat 2009). Macroadenomas start confined to the sella, the earliest extra-sellar extension is the upward growth into suprasellar cistern, this is anatomically explained by the hard-bony relationship in other directions. Less commonly macroadenomas are seen extending into the sphenoid sinus downwards, or laterally encasing the ICA within the cavernous sinus. (Ouyang, Rothfus et al. 2011)

Headache is the most common presentation of pituitary adenoma (Kim, Jin et al. 2019), in this study headache was the first primary presentation if hormonal presentations are separately listed, while hormonal presentation, in total, are of higher incidence. It's noted that patients with secretory adenomas seek medical advice in early stages due to hormonal related symptoms prior to visual pathway compression, except when amenorrhea is masked by menopause, Case 3 .

Though it's not the most common presentation, optic pathway compression is eventual when the tumor volume exceeds certain limit, functioning adenomas tend to present early with hormonal manifestations prior to visual presentation, this was also concluded by Brada et al (Brada, Rajan et al. 1993) who reported visual compression in $73 \%$ of non-functioning adenoma patients, while only in $44.7 \%$ in functioning ones. In the current study, examination showed 28 patients $(80 \%)$ of patients were found to have various degrees of visual impairment, taking in consideration that patients with history of errors of refraction and/or eye pathology were excluded, the incidence of visual impairment due to pituitary macroadenoma is higher than presentation which in this study was (20 patients, $57 \%$ ); with either main or secondary complaint.

Visual acuity deficits due to optic pathway compression by pituitary tumors are studied in several publications (Kim, Jin et al. 2019) (Kasputyte, Slatkeviciene et al. 2013) (Wang, Sun et al. 2008), as well as explained by the anatomical relationship of the pituitary gland to the optic pathway. In this study, the threshold of optic compression regarding volume and height of the tumor is identified.

Various anatomical factors may affect the degree of optic pathway compression including site of the chiasm and the capacity of suprasellar cistern which anatomically ranges from $8-13 \mathrm{~mm}$ (Rhoton 2002), this may explain the discrepancy between case 1 where the adenoma is greater in size and height than case 3 and yet shows radiologically less optic compression and clinically less visual impairment.

The volume of the pituitary tumor can be accurately measured via the traditional equation $1 / 2$ (length $x$ width $x$ height). This method was validated by Chi-Cheng Chuang et al (Chuang, Lin et al. 2017), where the equation was proven to be accurate for pre-operative assessment, but not valid for residual and hence recurrence, recurrent cases where excluded in this study based on that conclusion.

Similar to our results, $\mathrm{H}$ Wang et al (Wang, Sun et al. 2008) reported significant visual impairment in 
patients with Hardy's grade above B, with no significant difference between the groups B, C and D. These results raise the debate regarding various factors affecting chiasm compression course, as for $2 \mathrm{~cm}$ height i.e Hardy grade $B$ is the threshold for optic manifestations, lack of significant difference among greater sizes may be attributed to other factors including tumor consistency, growth rate and vascular interruption to the optic pathway. Anatomical reserve capacity and individual variation of cistern size should be taken in consideration. Visual field deficits, though not included in this study, are strongly associated with visual impairment in the majority (76 \%) of cases. (Wang, Sun et al. 2008)

Unlike previous studies, in this study, visual acuity of both patients' eyes were summed giving each patient a single value representing visual acuity, authors believe this is a more accurate assessment of overall visual impairment than listing each eye individually, since decussation of optic fibers takes place in optic chiasm which is the most common site of compression.

Visual acuity impairment is seldomly significant in pituitary microadeoma patients (less than $1 \mathrm{~cm}$ ) which are usually contained within the sella, while significant visual impairment starts beyond $2 \mathrm{~cm}$ height which can be explained by the growth into the supra-sellar cistern i.e transformation to Hardy grade B(Rilliet, Mohr et al. 1981).

According to Monteiro et al (Monteiro, Zambon et al. 2010), tumor size was the best predictor for postoperative visual recovery, the author reported that visual acuity is serviceable before optic atrophy develops hence the importance of correlating tumor volume to clinical visual impairment.

\section{CONCLUSION}

Pituitary macroadenomas with height greater than 2 $\mathrm{cm}$ and/or volume greater than $5 \mathrm{cc}$ are significantly associated with visual acuity impairment, behavior beyond these values (Hardy groups $B, C$ and $D$ ) is not linear and its course can neither be predicted nor explained by tumor size solely, to predict visual impairment due to pituitary macroadenomas, further multi-factorial studies are encouraged.

\section{DECLARATIONS}

Ethical approval: N/A, retrospective analysis of archive. Consent for Publication: N/A.

Availability of data and materials: please contact the author for data requests.
Competing interests: the authors declare that they have no competing interests.

Funding: No fund was received for this study. Authors' contribution:

ME: principle investigator, data collection, statistics and contribution to writing.

MS: data collection, revision.

MD: data collection, contribution to writing, revision and amendments.

Authors' information: all authors are staff members of neurosurgery department in Mansoura faculty of medicine and its affiliated hospital; Mansoura University Hospital.

\section{REFERENCES}

1. Asa, S. L. and S. Ezzat (2009). "The pathogenesis of pituitary tumors." Annu Rev Pathol 4: 97-126.

2. Brada, M., B. Rajan, et al. (1993). "The long-term efficacy of conservative surgery and radiotherapy in the control of pituitary adenomas." Clin Endocrinol (Oxf) 38(6): 571 578.

3. Chuang, C. C., S. Y. Lin, et al. (2017). "Different Volumetric Measurement Methods for Pituitary Adenomas and Their Crucial Clinical Significance." Sci Rep 7: 40792.

4. Cox, T. D. and A. D. Elster (1991). "Normal pituitary gland: changes in shape, size, and signal intensity during the 1st year of life at MR imaging." Radiology 179(3): 721-724.

5. Grover, S., G. A. Fishman, et al. (1999). "Visual acuity impairment in patients with retinitis pigmentosa at age 45 years or older." Ophthalmology 106(9): 1780-1785.

6. Jagannathan, J., A. S. Dumont, et al. (2005). "Genetics of pituitary adenomas: current theories and future implications." Neurosurg Focus 19(5): E4.

7. Kasputyte, R., G. Slatkeviciene, et al. (2013). "Changes of visual functions in patients with pituitary adenoma." Medicina (Kaunas) 49(3): 132-137.

8. Kim, T. G., K. H. Jin, et al. (2019). "Clinical characteristics and ophthalmologic findings of pituitary adenoma in Korean patients." Int Ophthalmol 39(1): 21-31.

9. Monteiro, M. L., B. K. Zambon, et al. (2010). "Predictive factors for the development of visual loss in patients with pituitary macroadenomas and for visual recovery after optic pathway decompression." Can J Ophthalmol 45(4): 404-408.

10. Munoz Negrete, F. J. and G. Rebolleda (2002). "[Automated perimetry and neuro-ophthalmology. Topographic correlation]." Arch Soc Esp Oftalmol 77(8): 413-428.

11. Ouyang, T., W. E. Rothfus, et al. (2011). "Imaging of the pituitary." Radiol Clin North Am 49(3): 549-571, vii.

12. Rhoton, A. L.,Jr. (2002). "The sellar region." Neurosurgery 51(4 Suppl): S335-374.

13. Rilliet, B., G. Mohr, et al. (1981). "Calcifications in pituitary adenomas." Surg Neurol 15(4): 249-255.

14. Wang, H., W. Sun, et al. (2008). "The pattern of visual impairment in patients with pituitary adenoma." J Int Med Res 36(5): 1064-1069. 